\title{
ON SIMULTANEOUS CHEBYSHEV APPROXIMATION IN THE "SUM', NORM
}

\author{
WILLIAM H. LING
}

ABSTRACT. Let $f_{1}, f_{2}$ be real valued functions on $[a, b]$ and let $S$ be a nonempty family of real valued functions on $[a, b]$. It is shown that the simultaneous approximation of $f_{1}$ and $f_{2}$ in the "sum" norm by elements of $S$ is, with one restriction, equivalent to the approximation of the arithmetic mean, $\left(f_{1}+f_{2}\right) / 2$. A complete characterization of best approximations in the "sum" norm is given including results for varisolvent families.

1. Introduction. Let $f_{1}, f_{2}$ be real valued functions defined on the nondegenerate compact real interval $[a, b]$. Let $S$ be a nonempty family of real valued functions on $[a, b]$. For any real valued function $f$ on $[a, b]$, $\|f\|$ shall mean $\sup _{x \in[a, b]}|f(x)|$. We are concerned with approximating $f_{1}$ and $f_{2}$ simultaneously in the "sum" norm by elements of $S$, i.e. we wish to minimize the expression $\left\|\left|f_{1}-s\right|+\left|f_{2}-s\right|\right\|$. If there exists an $s^{*} \in S$ such that

$$
\left\|\left|f_{1}-s^{*}\right|+\left|f_{2}-s^{*}\right|\right\|=\inf _{s \in S}\left\|\left|f_{1}-s\right|+\left|f_{2}-s\right|\right\|,
$$

we say that $s^{*}$ is a best simultaneous approximation to $f_{1}$ and $f_{2}$ in the "sum" norm. Also we say $s^{*} \in S$ is a best approximation to $\left(f_{1}+f_{2}\right) / 2$ from $S$ if

$$
\left\|\left(f_{1}+f_{2}\right) / 2-s^{*}\right\|=\inf _{s \in S}\left\|\left(f_{1}+f_{2}\right) / 2-s\right\| .
$$

There have been some recent papers on simultaneous approximation; see [3], [5] and [6]. In [5] and [6], $l^{p}$-simultaneous approximation on the direct sum $X \oplus Y$ is discussed, where $X$ and $Y$ are normed linear spaces. The "sum" norm defined above yields a norm on $C[a, b] \oplus C[a, b]$ but it is not discussed in [5] or [6]. In [3], the classical simultaneous problem is shown to be equivalent to approximating the arithmetic mean, $\left(f_{1}+f_{2}\right) / 2$, of $f_{1}$ and $f_{2}$ with an additive weight function. We show here that approximating in the "sum" norm is, with one restriction, equivalent to approximating the arithmetic mean.

Received by the editors November 26, 1973 and, in revised form, February 20, 1974 .

AMS (MOS) subject classifications (1970). Primary 41A45, 41A50.

Key words and phrases, Simultaneous approximation, "sum" norm, best approximation, arithmetic mean, varisolvent family. 
2. Theorem and discussion. Let $\rho_{0}, \rho$ and $y_{0}$ be defined as $\rho_{o}=\inf _{s \in S}\left\|\frac{f_{1}+f_{2}}{2}-s\right\|, \quad \rho=\inf _{s \in S}\left\|\left|f_{1}-s\right|+\left|f_{2}-s\right|\right\|$ and $y_{o}=\left\|f_{2}-f_{1}\right\|$. We then have the following.

Theorem. Let $\rho_{o}, \rho$ and $y_{o}$ be defined as above. Then it follows that (a) if $\rho_{0} \geq y_{0} / 2$, then $\rho=2 \rho_{0}$, and

(b) if $\rho_{0}<y_{0} / 2$, then $\rho=y_{0}$.

Proof. The proof is general, not specifying $\rho_{0} \geq y_{0} / 2$ or $\rho_{0}<y_{0} / 2$ until the end.

Claim 1. $\rho \geq 2 \rho_{o}$.

Proof. We have

$$
\left\|\frac{f_{1}+f_{2}}{2}-s\right\|=\left\|\left(\frac{f_{1}-s}{2}\right)+\left(\frac{f_{2}-s}{2}\right)\right\| \leq \frac{1}{2}\left\|\left|f_{1}-s\right|+\left|f_{2}-s\right|\right\| .
$$

Taking the infimum over $S$ on both sides of this last inequality yields $\rho_{0} \leq$ $1 / 2 \rho$.

Claim 2. $\rho \geq y_{\circ}$.

Proof. For any $s \in S$, we have

$$
\left|f_{1}(x)-s(x)\right|+\left|f_{2}(x)-s(x)\right| \geq\left|f_{1}(x)-f_{2}(x)\right|
$$

for all $x \in[a, b]$ which implies that $\left\|\left|f_{1}-s\right|+\left|f_{2}-s\right|\right\| \geq y_{0}$. Thus $\rho \geq y_{\circ}$.

Now recall that $|a+b|+|a-b|=2 \max \{|a|,|b|\}$ is an identity for all real numbers $a$ and $b$. Identifying $a$ with $\left(f_{1}(x)+f_{2}(x)\right) / 2-s(x)$ and $b$ with $\left(f_{1}(x)-f_{2}(x)\right) / 2$ we may write $\left|f_{1}(x)-s(x)\right|+\left|f_{2}(x)-s(x)\right|=2 \max \left\{\left|\frac{f_{1}(x)+f_{2}(x)}{2}-s(x)\right|,\left|\frac{f_{1}(x)-f_{2}(x)}{2}\right|\right\}$.

Taking the supremum over $x$ on both sides of this last equality yields

$$
\begin{aligned}
\left\|\left|f_{1}-s\right|+\left|f_{2}-s\right|\right\| & =2 \sup _{x \in[a, b]}\left[\max \left\{\left|\frac{f_{1}(x)+f_{2}(x)}{2}-s(x)\right|,\left|\frac{f_{1}(x)-f_{2}(x)}{2}\right|\right\}\right] \\
& \leq 2 \max \left\{\left\|\frac{f_{1}+f_{2}}{2}-s\right\|,\left\|\frac{f_{1}-f_{2}}{2}\right\|\right\} \\
& =2 \max \left\{\left\|\frac{f_{1}+f_{2}}{2}-s\right\|, \frac{y_{o}}{2}\right\} ;
\end{aligned}
$$

i.e. 


$$
\left\|\left|f_{1}-s\right|+\left|f_{2}-s\right|\right\| \leq 2 \max \left\{\left\|\left(f_{1}+f_{2}\right) / 2-s\right\|, y_{o} / 2\right\}
$$

Now taking the infimum over $S$ on both sides of this last inequality gives

$$
\begin{aligned}
\rho & \leq 2 \inf _{s \in S} \max \left\{\left\|\frac{f_{1}+f_{2}}{2}-s\right\|, \frac{y_{o}}{2}\right\} \\
& =2 \max \left\{\inf _{s \in S}\left\|\frac{f_{1}+f_{2}}{2}-s\right\|, \frac{y_{o}}{2}\right\}=2 \max \left\{\rho_{o}, y_{o} / 2\right\}_{0} .
\end{aligned}
$$

It follows that

$$
\rho \leq 2 \max \left\{\rho_{o}, y_{o} / 2\right\}
$$

The proof is now complete. For part (a) of the Theorem, if $\rho_{o} \geq y_{o} / 2$, inequality (A) combined with Claim 1 , ensures $\rho=2 \rho_{0}$. For part (b), if $\rho_{0}<$ $y_{o} / 2$, inequality $(A)$ combined with Claim 2, ensures $\rho=y_{0}$. Q.E.D.

The Theorem plus a short argument by contradiction gives the result that if $\rho_{o} \geq y_{o} / 2$, then $s^{*}$ is a best simultaneous approximation to $f_{1}, f_{2}$ in the "sum" norm iff $s^{*}$ is a best approximation to $\left(f_{1}+f_{2}\right) / 2$ from $S$. In a classical setting, one might have $f_{1}, f_{2}$ continuous on $[a, b]$ with $S$ a varisolvent family on $[a, b]$. In such a situation, if the best simultaneous approximation $s^{*}$ should exist, it would be unique; namely the unique best approximation to $\left(f_{1}+f_{2}\right) / 2$ from $S$. (For the definition of varisolvency see [7].)

On the other hand, if $\rho_{o}<y_{o} / 2$, the Theorem plus a short argument shows that if $B_{A}$ is the set of best approximations in the "sum" norm, i.e. if $B_{A}=\left\{s \in S:\left\|\left|f_{1}-s\right|+\left|f_{2}-s\right|\right\|=\rho\right\}$, then $B_{A}=\left\{s \in S: \|\left(f_{1}+f_{2}\right) / 2\right.$ $\left.-s \| \leq y_{o} / 2\right\}$. In particular, if $S$ is a varisolvent family and there exists an element $s^{*} \in B_{A}$ with $\left\|\left(f_{1}+f_{2}\right) / 2-s^{*}\right\|<y_{o} / 2$, varisolvency implies that $B_{A}$ contains an infinite number of functions.

Acknowledgement. The author wishes to thank Professor H. W. McLaughlin for a valuable remark.

\section{REFERENCES}

1. J. B. Diaz and H. W. McLaughlin, Simultaneous approximation of a set of bounded real functions, Math. Comp. 23 (1969), 583-593. MR 40 \#1733.

2. Simultaneous Chebyshev approximation of a set of bounded complexvalued functions, J. Approximation The ory 2 (1969), 419-432. MR 41 \#2026.

3. - - On simultaneous Chebyshev approximation and Chebyshev approximation with an additive weight, J. Approximation Theory 6 (1972), 68-71.

4. C. B. Dunham, Simultaneous Chebyshev approximation of functions on an interval, Proc. Amer. Math. Soc. 18 (1967), 472-477. MR 35 \#3334.

5. D. S. Goel, A. S. B. Holland, C. Nasim and B. N. Sahney, On best simultaneous approximation in normed linear spaces, University of Calgary, Research Paper No. 186, March 1973. 
6. D. S. Goel, A. S. B. Holland, C. Nasim and B. N. Sahney, Characterization of an element of best $l^{p}$-simultaneous approximation, University of Calgary, Research Paper No. 188, May 1973.

7. J. R. Rice, The approximation of functions. Vol. II: Nonlinear and multivariate theory, Addison-Wesley, Reading, Mass., 1969. MR 39 \#5989.

DEPARTMENT OF MATHEMATICS, UNION COLLEGE, SCHENECTADY, NEW YORK 12308 